\title{
Some new efforts concerning the photoelectric effect using the inverted new intensity formula and some efficiency raising mechanisms of solar cells
}

\author{
Bo Thelin \\ Solarphotonics HB Granitvägen 12B 75243 Uppsala Sweden
}

\begin{abstract}
In this paper new aspects concerning the current in the photoelectric effect are presented. By using the inverted form of the new intensity formula in optical emission spectroscopy, an explanation of many experimental current versus frequency observations are given. The ionization energy of many different elements has also shown to be important in the photo- electric effect in connection with this intensity formula.

This paper also shows some ways to increase the current from a solar cell by structuring the surface of the solar cell. Some physical effects which are dominant in accordance to this structuring, are discussed.

Keywords : photoelectric effect, electron spectroscopy, laser experiments solar cells, texturisation
\end{abstract}

\section{Introduction}

Concerning the fundamental laws of photo electricity it was discovered that the photoelectric current is directly proportional to the intensity of light. It was discovered that photons of sufficiently short wavelengths will ionize atoms and molecules when emitting a sample.

According to the Photoelectric Effect by Einstein , the kinetic energy of the electrons $\left(\mathrm{E}_{\mathrm{kin}}\right)$ equals the difference between then photon energy (hv) of the impacting photons and the ionization energy (I) of the target $\left(E_{k i n}=h v-I\right)$. It was also discovered a heating effect by the radiation and the consequently rise of the temperature of the absorbing material.

More exact methods involve measurements of the photoelectron current density $\mathrm{i}$ as a function of the irradiated frequency $v$. The dependence of the current $\mathrm{i}$ on the frequency $v$ can be represented by two different characteristic curves : the usual photo effect, which has an $\mathrm{e}^{v}$ - structure a bit in on the $v$-axis and the "selective" photo effect which starts in the same way as the normal one, but has a maximum in the photocurrent curve. The usual photo effect is occurring for most clean metal surfaces while the selective photo effect is working with thin films of alkali metals put on metallic backings as in photo cathodes.

There are some methods from the literature which try to explain the current versus frequency dependence. This dependence is seen and explained in different ways in the literature. Some of these are mentioned below.

The method of Fowler [1] is called the isotherm method of determining the work function from the spectral distribution curve of photo electron emission from a metal surface at a temperature $\mathrm{T}$. In his investigation the photocurrent $\mathrm{i} / \mathrm{T}^{2}$ is measured and plotted versus $\mathrm{h} v / \mathrm{k}$. This investigation gives for the first time a satisfactory theory of the effect of temperature on photoelectric emission together with the theory by Sommerfield [2].

In the Schottky theory[3] has been applies successfully to the problem of electron emission in accelerating fields. He has used a formula between current and the accelerating field and temperature.

In many other methods from the literature big discrepancies between experimental methods and theory have been appeared.

On the other hand, when using methods used in this paper, based on the new intensity formula, excellent agreement between theory and experiments have been held.

It has also been possible to increase the current in solar cells by structuring the surface(V-form) of the solar cell. This has been demonstrated in many papers which has caused better efficiency for such solar cells. This paper also demonstrates similar results which have increased the current and the power from the solar cell. New ideas and physical effects concerning the V-form of solar cells are also presented.

\section{This photoelectric investigation}

In this very paper new results have been obtained by using the new intensity formula in optical emission spectroscopy. By using the inverted form of this formula good correlation between the current and frequency has been obtained from photoelectric experiments at different field strengths. At photoelectric and thermionic experiments at different elements the importance of the ionization energy in the photoelectric effect has been demonstrated. 


\subsection{The new intensity formula}

The new intensity formula has recently been published in two open access summary papers [ 4 ] [ 5 ] of this project and the new strong proofs from the literature of this formula. This new formula has earlier been verified in many experimental papers, which are earlier summarized in [6] and [7] and also in a new fundamental basic theory [8].

According to the new theory, the intensity I of a spectral line is given by

$$
I=\mathrm{C} \lambda^{-2} \exp \left(-\mathrm{J} / \mathrm{kT}_{\mathrm{e}}\right) /\left(\exp \left(\mathrm{hv}_{\mathrm{k}} / \mathrm{kT} \mathrm{T}_{\mathrm{e}}\right)-1\right)
$$

where $\mathrm{J}$ is here the ionization energy, and $\mathrm{C}$ is a factor given by transition probabilities, number densities and sample properties. $\lambda$ and $v$ are here wavelength and frequency of the atomic spectral line and the temperature $\mathrm{T}_{\mathrm{e}}$ is the electron temperature.

This means that the new intensity formula consists of 4 parts : the C-factor, the $\lambda^{-2}$-part,the $\mathrm{J}$-dependence e $\mathrm{x} p$ $\left(-\mathrm{J} / \mathrm{k} \mathrm{T}_{\mathrm{e}}\right)$ and the Planck factor $1 /\left(\mathrm{e} \mathrm{xp}\left(\mathrm{h} v / \mathrm{k} \mathrm{T}_{\mathrm{e}}\right)-1\right)$

As this intensity formula deals with number of emitting photons from a plasma, it has also shown to be possible to use it on the reverse process, where incoming photons emit electrons into a current.

An experimental proof of that is shown below.

The fact which is important in this very paper about the photoelectric effect is a relationship between current and frequency. The usual appearance from the literature of this experimental

relationship is an $\mathrm{i}=\mathrm{e}^{\mathrm{v}}$-structure , where the current (y-axis) is an exponential function of the frequency(xaxis), and starts from the $v_{0}$ (threshold) a bit in on the $v$-axis. This experimental fact is what the different theories in the introduction have tried to fit, with some difficulties according to their own opinions.

\subsection{The inverted intensity formula}

On the other hand, by using equation 1 and express the $\mathrm{C}$-factor as approximately

$$
\mathrm{C}=\mathrm{I} \lambda^{2} \exp \left((\mathrm{J}+\mathrm{hv}) / \mathbf{k T} \mathrm{T}_{\mathrm{e}}\right)
$$

a very good fit could be obtained. The $\mathrm{C}$-factor is here proportional to the current in the photoelectrical effect. Such a nice fit can be seen in Fig 1, from a photoelectrical experiment

with potassium at different field strengths. In this fit current ratios have been calculated according to equation 2 (where the current $\mathrm{i} \alpha \mathrm{C}$ ).In this way only the $v$-dependence of the C-factor(current) appeared more clearly. This data were coming from Fig 3-46 p.111 of [9] and is demonstrating the influence of electric field on the photoelectric threshold of thin layers of potassium on tungsten.

\subsection{Experimental proof of the current versus frequency relationship}

This data current ratios were calculated from the data and compared with the current from equation 2. This can be seen in equation 3 .

$$
\begin{aligned}
& i_{k} / i_{k-1}=\left(\exp \left(h v_{k} / k T_{e}\right)-1\right)\left(h v_{k-1}\right)^{2} /\left(\exp \left(h v_{k-1} / k T_{e}\right)-1\right)\left(h v_{k}\right)^{2} \\
& \approx\left(v_{k-1} / v_{k}\right)^{2} \exp \left(h / k T_{e}\left(v_{k}-v_{k-1}\right)\right)
\end{aligned}
$$

Nice correlation between experimental and theoretical values were obtained at two different field strengths ( A and $\mathrm{B})$ in these experiments.

Similar graphs to Fig 1 can also be seen in many graphs in [9] and in photo detachment experiments in Fig 1.25 p.39 of [10] -and solar cell experiments Figs 19,25 and 27 of p.288,293 and 294 of [11], where photons create electrons into a current.

This $\mathrm{e}^{\mathrm{x}}-$ structure is very common in many graphs of [9] where the low frequency side goes up to a maximum. The high frequency side represents an $\mathrm{e}^{1 / \mathrm{x}}$ - structure. This can be seen in Fig 3-6 p.46 in [9]. This $\mathrm{e}^{1 / x}$-structure is probably caused by the heating effects of the sample. Therefore the $\mathrm{kT}_{\mathrm{e}}-$ part of equation 2 will be dominating the structure of the high frequency part and show a $\mathrm{e}^{1 / \mathrm{x}}$-structure.

These $\mathrm{e}^{\mathrm{x}}$ and $\mathrm{e}^{1 / \mathrm{x}}-$ structures are very common in [9] and in the literature of electron spectroscopy.

For example in papers which calculate the photo ionization cross section cross section Fig 4 p.360 and Fig 5 p.361 in vol 1 of (12) and Fig 21 p.172 of vol 5 of (12).

\subsection{The J-dependence of the photoelectric effect}

The influence of the J-term of equation 2 on the photoelectric effect, has also been seen in several ways. In Table 3-2 p.75-76 of [9] photoelectric and thermionic work functions of different elements have been studied. This table has been used in Fig 2, where the ionization energies(J) have been plotted versus the threshold wavelengths of that table. Here is it possible to study the photoelectric effect of different elements. Fig 2 is similar to Fig 1 if $\mathrm{i} \alpha \mathrm{C} \alpha \mathrm{J}$ according to equation 2 but include information from 22 different elements. Fig 2 is a clear evidence of $\mathrm{J}$-dependence of the photoelectric effect, where the slope of the curve increases with higher $(\mathrm{J}+\mathrm{h} v)$ - values $(5.5-16 \mathrm{eV})$ according to equation 2 . 
According to equation $2 \mathrm{~J} \alpha \mathrm{i}$ (current) which means that Fig 2 show the usual $\mathrm{e}^{v}$-dependence between the current and frequency as in Fig 1.

If we express $J$ as a function of $\lambda$ from equation 2 we obtain :

$$
\mathbf{J}=\mathbf{f}\left(\lambda_{0}\right)=\mathrm{k} \mathbf{T}_{\mathrm{e}} \ln \left(C / I \lambda^{2}\right)-\operatorname{hc} / \lambda
$$

We can see that the curve of Fig 2 has a typical 1 n-structure supporting equation 2.

The influence of $\mathrm{kT}_{\mathrm{e}}$ will increase for lower wavelengths of Table 3-2 in [9]. Therefore the slope of the low wavelength side is much higher than the high wavelength side of the curve. This is also seen by derivating this expression.

The derivative of equation 3 is :

$$
\mathbf{J} '=k \mathbf{T}_{\mathrm{e}}(\mathrm{I} / \mathrm{C})(-2 / \lambda)+\mathrm{hc} / \boldsymbol{\lambda}^{2}
$$

It is obvious here that $\mathrm{kT}_{\mathrm{e}}$ is very important for the slope of the curve. At lower wavelengths the $\mathrm{kT}_{\mathrm{e}}$ is higher which increases the slope of the curve. The heating effect at higher frequencies is a dominating part of the current versus frequency curve, mentioned above, which means that we obtain a maximum of the curve. In Fig 2 we can se that in the "knee" of Fig 2.

We can also study the threshold wavelength $\lambda_{0}$ as a function of $\mathrm{J}$. By using the expression by Einstein in the photoelectrical effect, mentioned in the introduction, where the kinetic energy of the electron is the difference between photon energy and the ionization energy, it is obvious that the photon energy at the threshold equals the ionization energy $\left(h v_{0}=\mathrm{J}\right)$.

By using this in equation 4 the following expression is held:

$$
\lambda_{0}=(\mathrm{C} / \mathrm{I})^{1 / 2} \exp \left(-\mathrm{J} / \mathbf{k} \mathbf{T}_{\mathrm{e}}\right)
$$

which is also in accordance with the results in Fig 2 for the metals.

Similar results to Fig 2 have also been seen for 19 different gases in Fig 3 from data of Table 7-5 p.274[9]. The wavelengths of the thresholds of this graph are situated in the ultraviolet range. The curve profile looks similar to Fig 2, but do not have the "knee" of Fig 2, probably because the fact that the heating effect is not so dominating in the gas phase here.

Similar graphs were also done for the alkali metals from Table 7-1 p. 250 in[9] and are shown in Fig 4. In this graph $\mathbf{J}$ was plotted versus series limit and seem to follow the appearance of Fig 2 and 3.

Another way to show that equation 2 seems to be important here, is the use Table 12-1,p.423 and Fig 12-8, p.424 in[9] for the alkali metals. These results are shown in Fig 5. The current here seems to follow $(\mathrm{J}+\mathrm{h} v)$ according to equation 2 and give a linear relationship. In these experiments with the alkali metals the sample was well prepared and the equipment was well outgased to obtain good results.

A similar experiment to Fig 2 for metals of very high temperatures is shown in Fig 6. In this graph a "knee" is shown similar to Fig 2 and the data comes from Table 3-1,p.45 in[9].

Equation 2 is also supported by newer breakdown experiments using lasers. This is illustrated in Fig 9.8 ,p.735, Figs $9.11,9.12$ and 9.13 ,p.744,745 in [10] where the laser intensity was plotted versus the breakdown pressure of the gas. These graphs have an almost negative linear structure.

The intensity of the laser beam is proportional to the temperature of the gas and evidently also proportional to the ionization grade $\left(\mathrm{n}^{+} / \mathrm{n}\right)$. This means that the intensity is proportional to

exp- $\left(\mathrm{J} / \mathrm{kT}_{\mathrm{e}}\right)$ according to the Saha equation and equation 1 for ions. As the graphs show a negative linear shape means that the breakdown pressure is proportional to $\exp \left(\mathrm{J} / \mathrm{kT}_{\mathrm{e}}\right)$ according to equation 2.

The $\mathbf{J}$ - term of equation 2 can also be shown when comparing the big difference in ionization energy between $\mathrm{A}(15.76 \mathrm{eV})$ and $\mathrm{He}(24.58 \mathrm{eV})$. This is also illustrated in the breakdown experiments with lasers(discussed above) and also in Fig 9.10, p.739 and Fig 9.13, p.745 in [10]. In these experiments a big separation between the $\mathrm{A}$ and $\mathrm{He}$-curves is shown, where the He-curve is situated at much higher pressure. This is in accordance to equation 2. This $\mathbf{J}$-dependence is also illustrated in the laser experiments in Fig 9.9, p.737 in [10], where the elements with higher ionization energy are situated at higher pressure according to equation 2.

\section{Some physical effects increasing the efficiency of solar cell arrangements.}

In this investigation different arrangements of solar cells have been performed to increase the total efficiency of this arrangement. The main issue here is to the use the depth of the solar cell arrangements, where the solar cells are forming some kind of structured formation. In this way it has shown to be possible to get advantage of some more physical principals compared to flat arrangements.

\subsection{The V-structure}

In this experiment two solar cells are put together in a V-structure with an angle $\mathrm{v}$ between the solar cells. These solar cells were Si-cells with the same reflectivity. The solar cells were connected in a serie and it was possible to measure both the voltage and the current over the solar cells simultaneously with an ampere meter and a voltmeter instruments. The same measurements were done on a flat arrangement $\left(\mathrm{v}=180^{\circ}\right)$ of these 
solar cells. An 1kW-lamp was used which illuminated the solar V-and the flat solar cell at the distance of 60 $\mathrm{cm}$. The results from these measurements can be seen in Fig 7, where the current/inlet area

was plotted versus the voltage for both $\mathrm{V}$-stucture and flat structure arrangements. The result of this figure is an relative increase of around $60 \%$ for the power maximum/inlet area $\left(\mathrm{P}_{\max }=\mathrm{U} \times \mathrm{I}\right)$ for the V-structure compared to the flat case when looking on the "knee" of the diagram.

This increase was more than $60 \%$ when Si-cells of high reflectivity were used when CIS-solar cells(around 90 $\%)$ were studied.

Similar investigations have been done by several investigators [13-16].

In this investigation the power/inlet area was studied and plotted versus the V-angle(v). This is illustrated in Fig (8) where two different physical effects are illustrated.

The first effect is the multi reflection where photons are reflecting between the V-sides. This means that photons have bigger chance of being absorbed by the solar cell surface. This effect has the biggest value at an angle of $38^{\circ}$. This is illustrated in Fig( $(8)$, where

the area A illustrates the area of the graph which is most dependent of the multi reflection effect. This effect is mostly dominating in angles between 38-180 degrees. In the B-area

between $0-38$ degrees two physical effects is dominating : multi reflection and sigma-

effect $(\sigma)$ (surface charge density) in the solid state material. This effect is described in detail in [17], where the charge density $\rho$ is exponentially increasing with decreasing angle $v$ of the $V$-structure according to equation 7 . For the smallest angles this sigma effect is the most dominating effect.

$$
\sigma=Q\left(\frac{1}{v}\right) \rho^{(\pi / v)-1}
$$

where $\mathrm{Q}=$ constant. This means that for an angle of $38^{\circ}(\pi / \mathrm{v} \sim 5)$, which means $\sigma \sim \rho^{4}$. This means still higher current densities at lower angles.

\section{Discussion}

The results in this paper explain the general appearance of the shape of the current versus frequency expression in the photoeffect. On the low frequency side of the curve, an $\mathrm{e}^{\mathrm{x}}$ - structure is appeared, and on the high frequency side an $\mathrm{e}^{1 / \mathrm{x}}$ - structure is appeared. This appearance is general for all kinds of current measurements from old and newer papers in the literature. These observations have also been seen in the electron spectroscopy field where the photoionization cross section has been calculated versus photon energy. These graphs have also the same $\mathrm{e}^{\mathrm{x}}$ and $\mathrm{e}^{1 / \mathrm{x}}$ - structure as is described above. One explanation of this $\mathrm{e}^{\mathrm{x}}-$ structure is the photon energy hv of equation 2 and the $\mathrm{e}^{1 / \mathrm{x}}$-structure depends on the heating effect $\mathrm{kT}_{\mathrm{e}}$ which raises with increasing photon energy. Fig 1 is a strong evidence of the frequency depending part of equation 2 .

Fig 2 is a strong evidence of the J-term of equation 2.From this graph it is possible to study the J-dependence of 22 different elements(metals). The "knee" of this curve depends of the heating effect at higher frequencies, which is also clearly demonstrated by the derivative

in equation 5. The $\mathbf{J}$ - dependence is also demonstrated in Fig 3 for 16 different gases. The threshold wavelengths here are much shorter compared to Fig 2 and the heating effect is

lower. A similar graph to Fig 2 is seen in Fig 6, where the metals were heated to high temperatures. This graph also demonstrates a clear "knee" at high temperatures with more spread in the experimental points.

Alkali metals were also studied in a similar graph to Figs 2,3 and 6 and is seen in Fig 4. The heating effect here is not so high and the general appearance is the same as the other graphs.

In this data it was possible to plot $\ln \left(\mathrm{i} / \lambda^{2}\right)$ versus $(\mathrm{J}+\mathrm{h} v)$ according to equation 2 ,

where $\mathrm{i} \alpha \mathrm{C}$ is the current (number density of electrons). This graph is seen in Fig 5 is linear

and supports equation 2 . The $\mathbf{J}$-term in equation 2 is also supported by different breakdown experiments with lasers. In these experiments the electrons were created with the photoeffect

from strong laser beams. The breakdown pressure is here proportional to the number density

of electrons(current) and therefore it will follow equation 2.

As many papers in the past have shown, this paper also shows enhancements of the currents from solar cells by structuring the surface (V-form) of the solar cells. This also means higher efficiencies for solar cell systems. This paper also discuss two fundamental physical effects which are dominating the V-structure at different angles. The equation 7 is an expression of the surface charge density for V-structures of metals. Solar cells have great similarities with metals when they are heavily illuminated. Therefore it is plausible to use equation7 to explain the big enhancements of the current in solar cells, which are observed when the V-angle is decreased ( less than $38^{\circ}$ ). This fact can also be an explanation of the big current enhancements observed in black silicon solar cells. These big enhancements can be read about on the internet (Prof. E.Mazur from Harward University, Black Silicon). The micro structure of these black silicon solar cells is a forest of rods fabricated by a laser. The $\mathrm{V}$-angles here are naturally very small which give high charge density according to equation 7 and evidently also higher currents. Any earlier physical explanations of these big current enhancements have not yet come up until now. 
There is a believe from many people in this field that structured solar cells have a big future.

\section{Acknowledgement :}

I express a big gratitude to my friend and collegue Dr. Sten Yngström at the Swedish Institute of Space Physics for inspiring and skillful cooperation during many years. I also express gratitude to my collegues civ.ing. Einar Söderman and ing.Zahm Ottoson at Uppsala University for technical assistance and advise in the technical parts of this work.

Fowler R. H. , Phys.Rev. 38 , 45 ( 1931)

\section{References:}

[2] Sommerfeld A., Zeits. f. Phys. , 47, $1,(1928)$

[3] SchottkyW., Zeits. f. Phys., 14, 63, ( 1923 )

[4] Thelin B. , Eurasian J. Anal. Chem. 4, Nr 3, 226 , (2009)

[5] Thelin B., African Physical Review, 4 , 121-128, (2010)

[6] Yngström S. and Thelin B. , Appl. Spectrosc. 44, 1566 ( 1990)

[7] Thelin B. , Appl. Spectrosc. 44, $818,(1990)$

[8] Yngström S. , Internat. J. Theoret. Phys. 33, nr 7, 1479, ( 1994)

[9] Hughes A.L. and DuBridge L.A., Photoelectric Phenomena, 1932

[10] Meek J.M. and J.D.Craggs J.D. , Electrical Breakdown of Gases , 1978

[11] Tsubomura H. and Kobayaski H., Critical Rev. in Solid State and Material Sciences, 18 (3), 261 - 326 ( 1993 )

[12] Brundle C.R. and Baker A.D., Electron Spectroscopy Theory, Techniques and Application vol 1, 1977 and vol 5 , 1984

[13] Campell P. and Green M.A, J. Appl. Phys., 62 ,243, (1987)

[14] Campell P., Sol.Energy Matter., 21 , 165, 1990

[15] Jooss W., Fath P., Bucher E., Roberts S. and Bruton T.M., $17^{\text {th }}$ European Photovoltaic Solar Energy Conference, 22 -26 October 2001, Munich, Germany. Process Developmant for large area buried contact solar cell on multicrystalline silicon.

[16] Terheiden B., Fische B., Fath P. and Bucher E. , $17^{\text {th }}$ European Photovoltaic Solar Energy Conference, $22-26$ October 2001 , Munich, Germany. Highly efficient mechanically V-textured silicon solar cells applying a novel shallow angle contacting scheme.

[17] Jackson J.D. , Classical Electrodynamics , 3: rd edition, p.78, 1989.

\section{Figures}

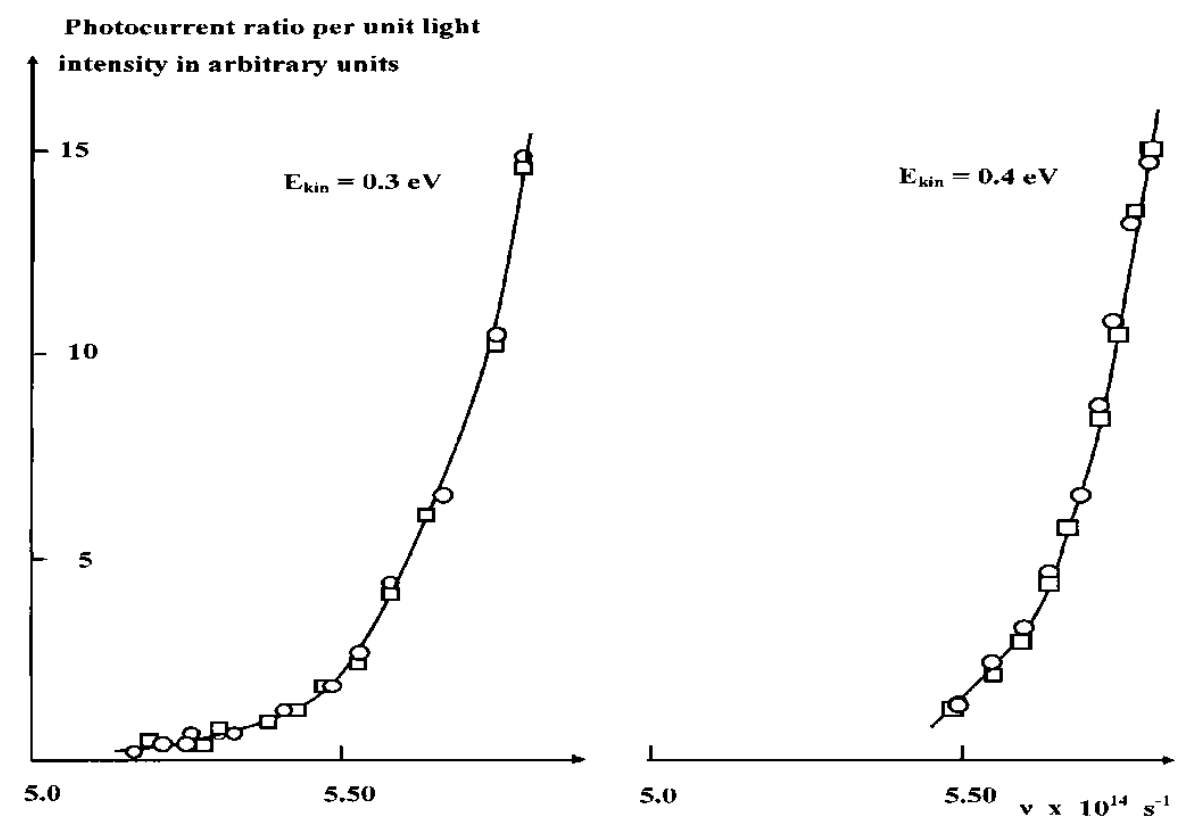

Fig 1

The $v$ - dependence of the photocurrent ratios between literature experiment(circles) and the new theory (squares)(inversed intensity formula) Results at different electron energies are shown 


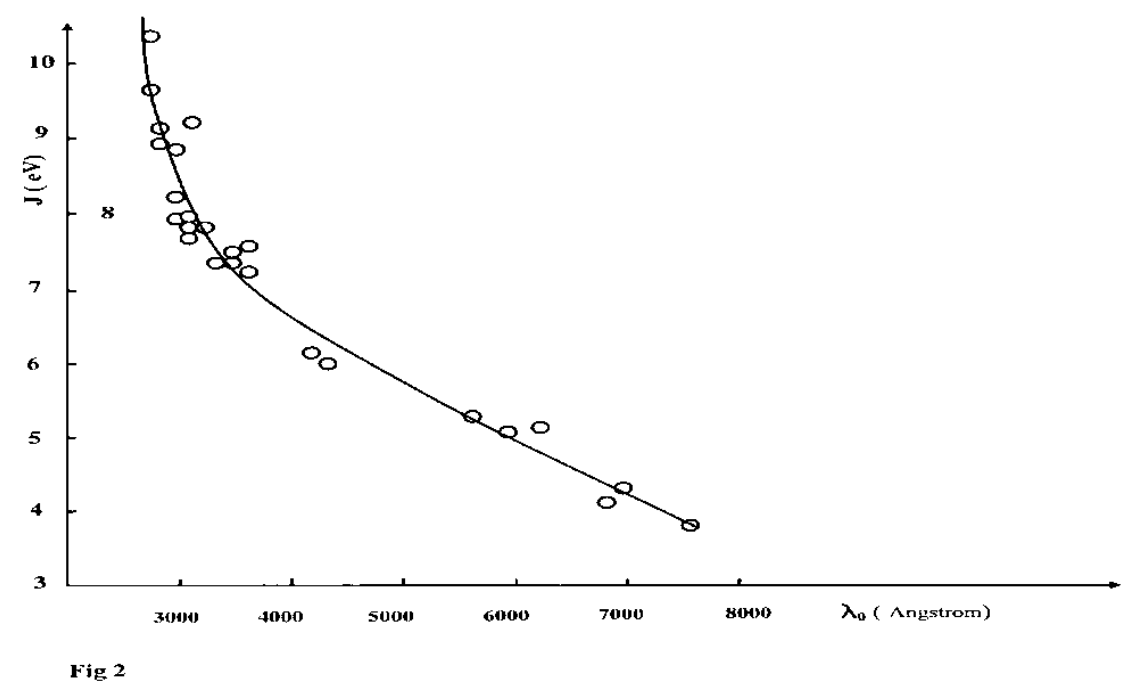

The influence of the ionization energy J versus the threshold wavelengths of the photoelectric effect. 22 different elements(metals) have been studied.

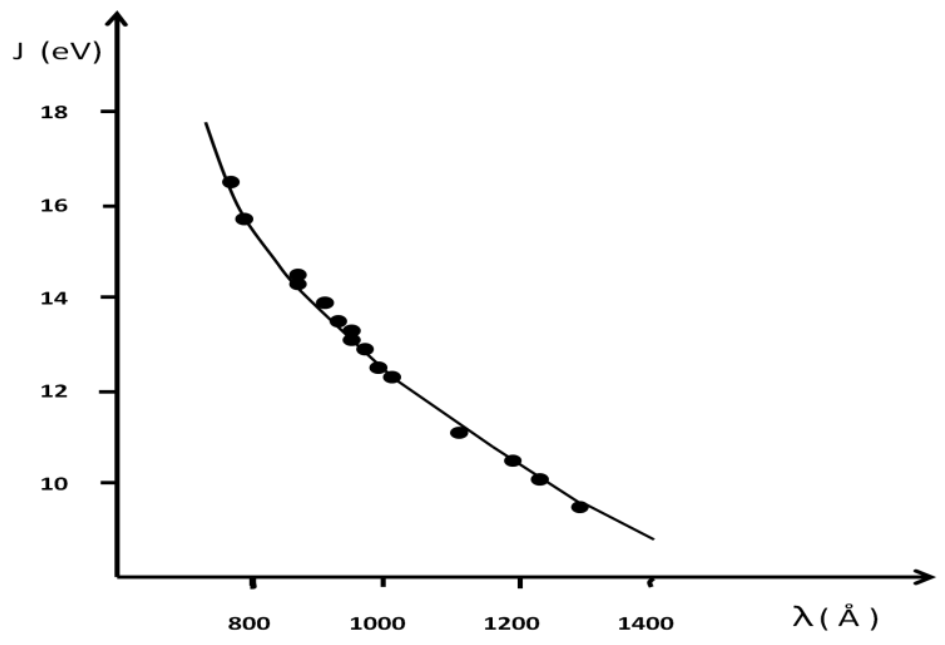

Fig 3The influence of the ionization energy $\mathrm{J}$ versus the threshold wavelengths of the photoelectric effect. 19 different gases have been studied.

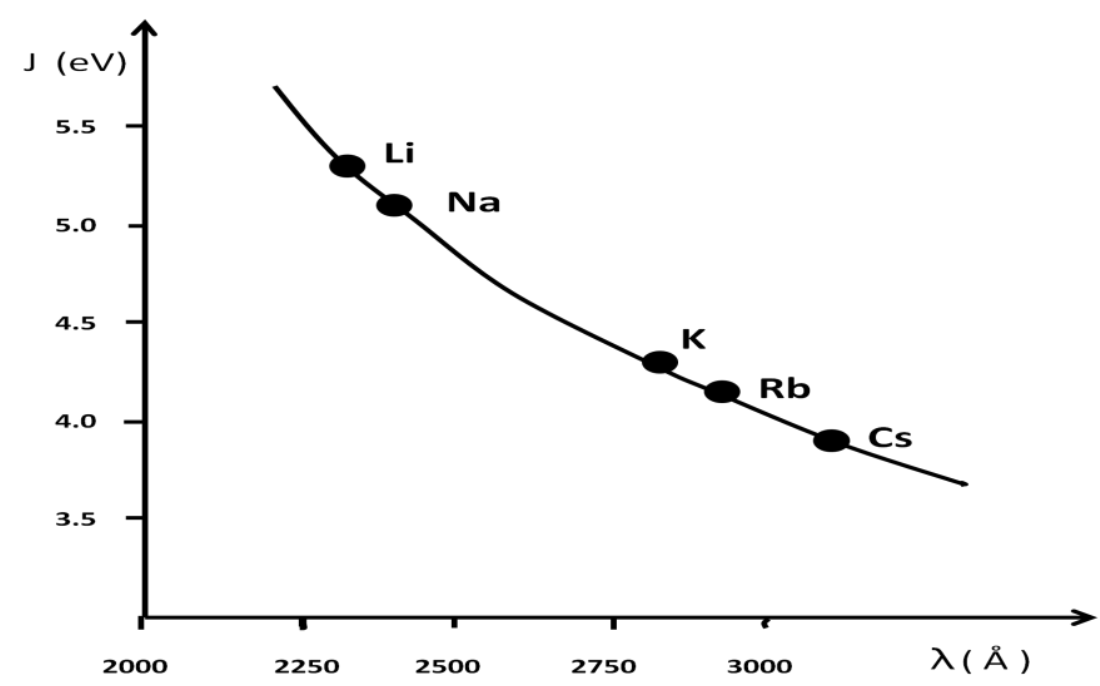

Fig 4The influence of the ionization energy $J$ versus the threshold wavelengths of the photoelectric effect. The alkali metals have been studied. 


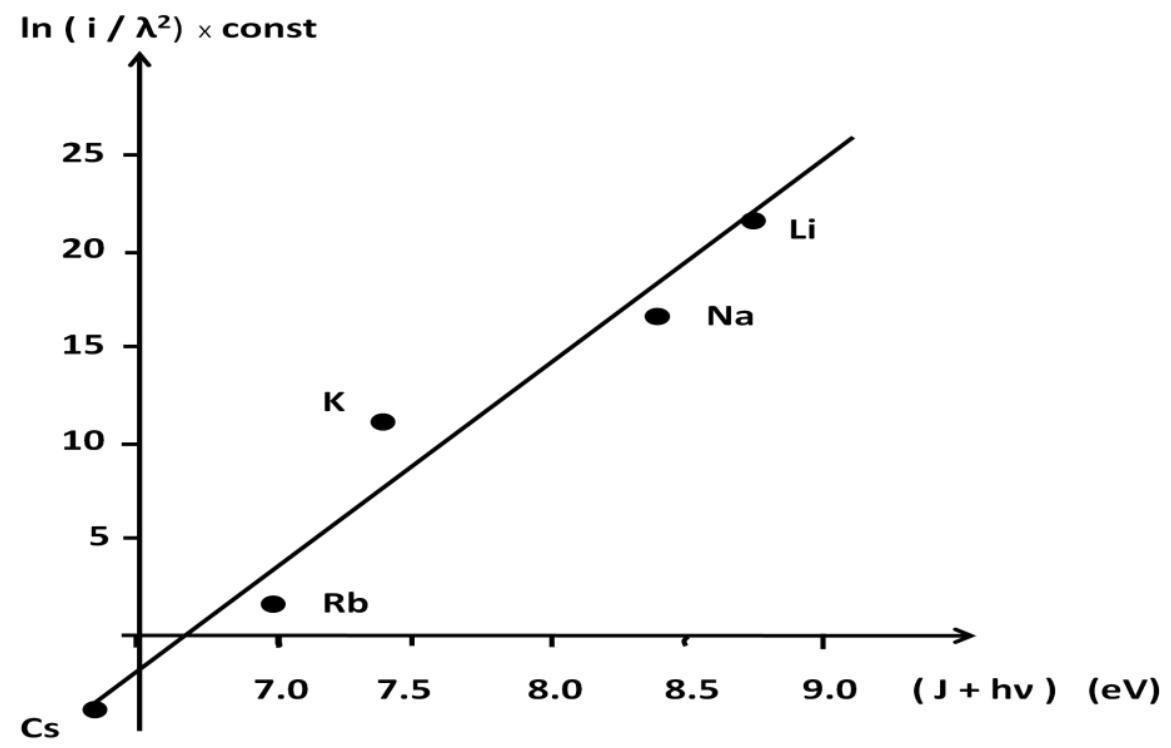

Fig 5 Linear relationship between current and $(\mathrm{J}+\mathrm{h} v)$ according to equation 2. Alkali metals were studied.

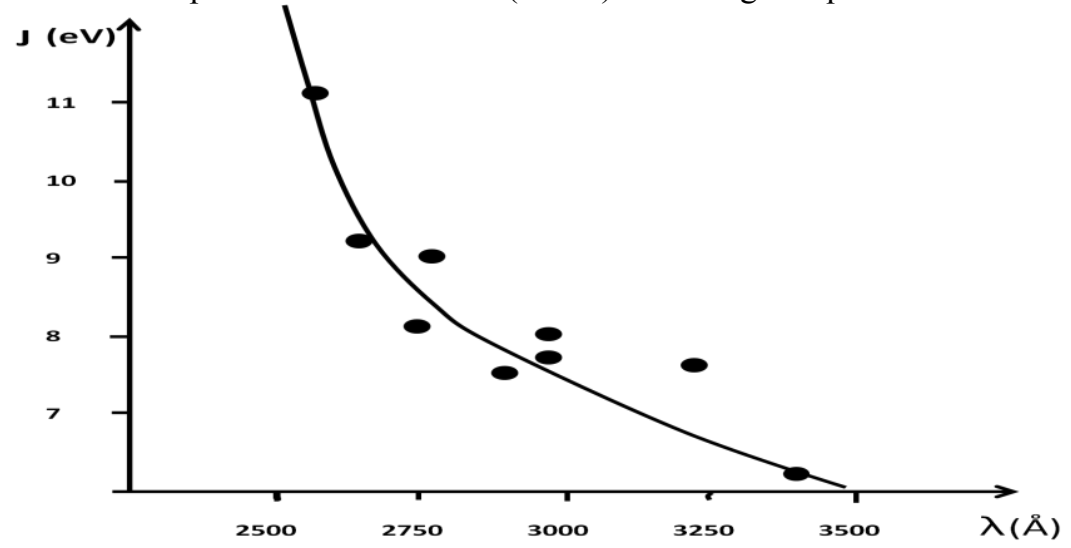

Fig 6 The influence of the ionization energy $\mathrm{J}$ versus the threshold wavelengths of the photoelectric effect. 9 different metals have been studied at high temperatures.

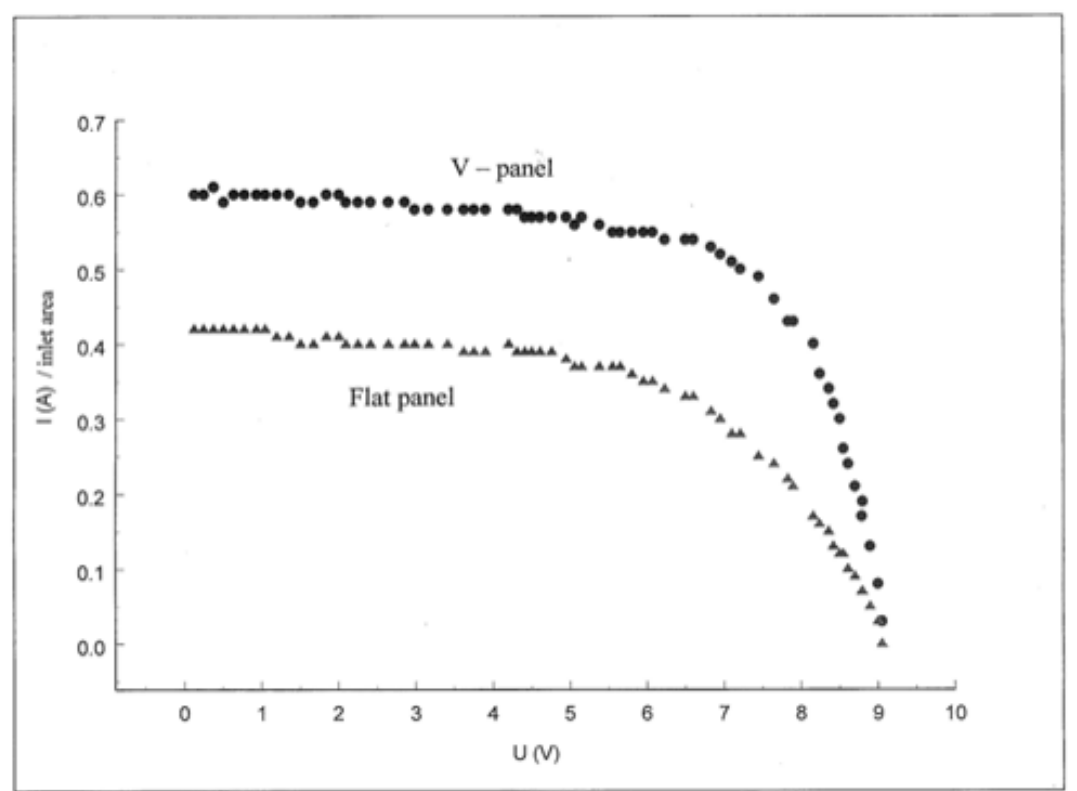

Fig 7 The current / inlet area versus voltage is here studied at a solar cell experiment.A V- structure arrangement and a Flat- structure arrangement are compared. 


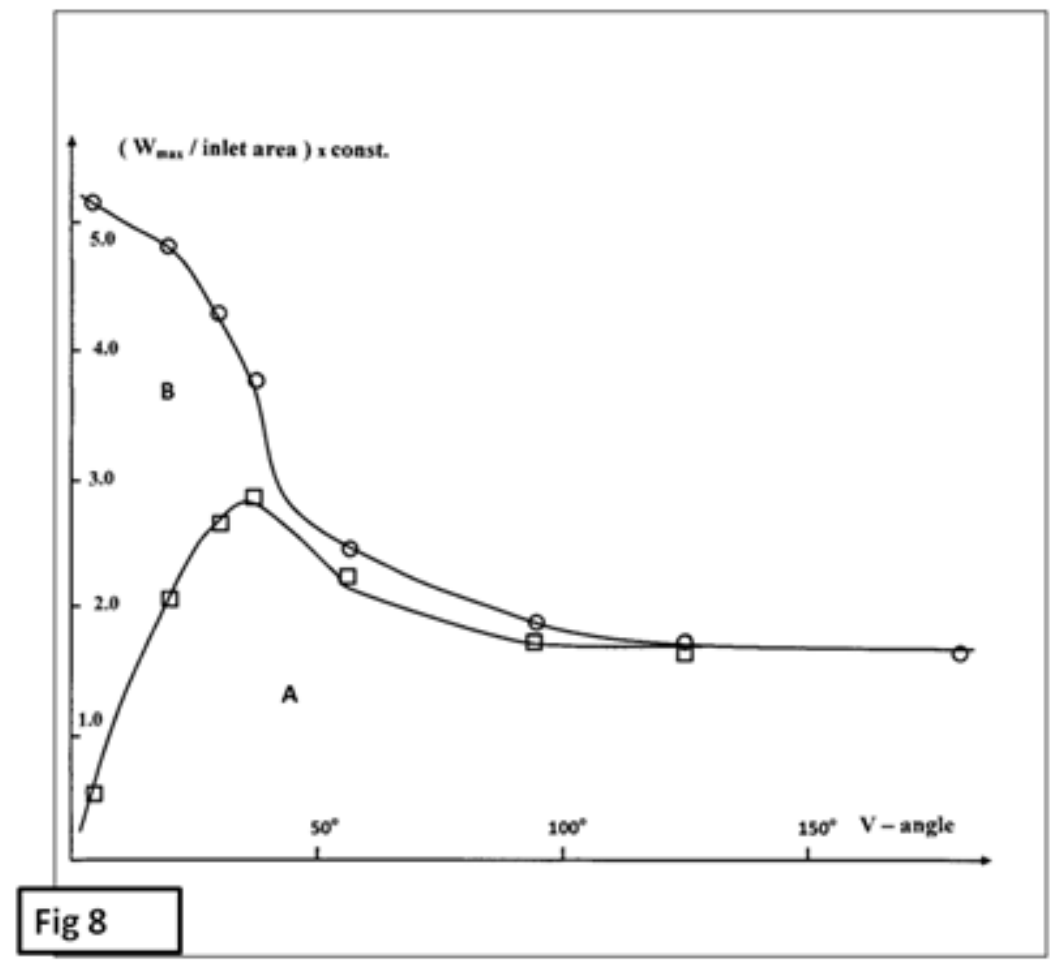

Fig 8 Power / inlet area versus v-angle is here studied for a V-structure solar cell Arrangement. In the A-area $\left(38^{\circ}-180^{\circ}\right)$ multi reflection (unfilled squares) is the dominating effect. In the B-area $\left(0^{\circ}-38^{\circ}\right)$, multireflection and $\sigma$-effect (unfilled circles) are the most dominating effects. 